\title{
Visualizing 3D Time-Dependent Foam Simulation Data
}

\author{
Dan R. Lipşa ${ }^{1}$, Robert S. Laramee ${ }^{1}$, Simon Cox $^{2}$, and I. Tudur Davies ${ }^{2}$ \\ 1 Visual and Interactive Computing Group, Department of Computer Science, \\ Swansea University, Swansea, UK \\ 2 Institute of Mathematics and Physics, Aberystwyth University, Aberystwyth, UK
}

\begin{abstract}
Liquid foams have important practical applications in mineral separation and oil recovery. However, the details of the foam mechanics in these applications are poorly understood. Foam scientists have used 2D foam simulations to model foam behavior and $2 \mathrm{D}$ visualization solutions have helped them explore and analyze their data. Three-dimensional foam simulations remove some of the simplifying assumptions made in $2 \mathrm{D}$ so they should provide better approximations of reality. Yet no foam specific $3 \mathrm{D}$ visualization tools exist. We describe a software tool for the exploration, visualization and analysis of time-dependent 3D foam simulation data. We present feedback from domain experts and new insights into foam behavior obtained using our tool.
\end{abstract}

\section{Introduction and Motivation}

Liquid foams have important practical applications which include mineral separation. Metals and valuable minerals are separated from rock by passing the ground ore through a foam which carries and collects the minerals for further processing. The efficacy of the separation process depends inter-alia on how objects with different size, shape and weight behave in a foam. A simulation related to this application is performed by scientists and used as case study in this work.

A liquid foam is a two-phase material consisting of gas bubbles separated by a continuous liquid network [18]. Liquid foams have a complex time-dependent behavior under stress that is not fully predictable. Foams behave like elastic solids for small deformations but when strain is increased they start behaving like viscous fluids. At high strain significant challenges arise because continuous changes in bubble shape and/or size can trigger discontinuous events in which the liquid network is rearranged (topological changes). This discontinuous temporal behavior at a small (bubble) scale creates difficulties in describing foam at a large scale, as a continuous medium. The main goal of foam research is to characterize foam behavior from measurable foam properties such as bubble size and its distribution, liquid fraction and surface tension.

A possible approach to study foam dynamics is to simulate foam rheology at the bubble scale, where scientists can choose a set of foam parameters and study the resulting foam behavior. Surface Evolver (SE) [2] is the standard tool for bubble-scale foam simulations with high accuracy in terms of static structure and quasi-static flow. 
Foam scientists use 2D foam simulations to model foam behavior; foam visualization solutions $[5,10]$ have helped them gain insights into their data. A $2 \mathrm{D}$ foam can be created experimentally by squeezing bubbles between parallel glass plates [15], thus providing a means to validate simulations. However, most real foams are 3D. Two-dimensional foam simulations might introduce additional errors and $2 \mathrm{D}$ foam experiments suffer from effects such as wall drag. Foam scientists would like to evaluate 3D foam simulations and assess and analyze differences between 2D and 3D simulations, but few visualization solutions exist for 3D foam simulation data.

Three-dimensional SE foam simulations present significant visualization and analysis challenges to researchers. Parsing is required for accessing simulation data and domain specific knowledge is required to deduce missing simulation attributes. Data is unstructured (polygonal bubbles), multi-attribute and timedependent. Large fluctuations in the simulation attributes are caused by bubble rearrangements. This means that general foam behavior is difficult to infer from individual time steps.

These challenges make it difficult to use a general purpose tool to visualize and analyze foam simulation data. Domain-experts analysis and visualization methods only partially address these challenges. They require intervention in the simulation code to summarize and save data and may require re-running the simulation if different data needs to be saved. Scientists use available tools for generating plots of the data but these tools do not enable interaction with the data and do not facilitate comparison of datasets.

Our work is a design study. We describe visualization solutions that address foam research challenges. Our software complements the tools and methods used by domain scientists to provide new ways to interact with and visualize foam simulation data. To the best of our knowledge, our software is the first comprehensive visualization solution for 3D foam simulation data modeled with Surface Evolver.

The rest of the paper is organized as follows: Sec. 2 presents related work. Solutions to explore, visualize and analyze foam simulation data are described in Sec. 3. We present several different examples of their use in Sec. 4 and end with conclusions and future work in Sec. 5 .

\section{Related Work}

Computer graphics researchers are interested in rendering soap bubbles $[6,7,17]$, foams [13] and water sprays [12], however, they render the appearance of natural phenomena while avoiding the large computational cost of physically-accurate simulations. Most work in the visualization literature [11] deals with visualization of static foam or foam-like structures $[1,8,9]$. Existing tools to manipulate Surface Evolver data include evmovie, which is distributed with Evolver, and the Surface Evolver Fluid Interface Tool (SE-FIT) [4]. Evmovie scrolls through a sequence of evolver files, while SE-FIT provides a graphical interface for interacting with Surface Evolver. In previous work we $[5,10]$ presented a tool for exploration, visualization and analysis of foam simulation data in $2 \mathrm{D}$, and here we extend its functionality to $3 \mathrm{D}$. 


\section{Visualization and Interaction}

Our visualization solutions are driven by the foam research challenges listed in Sec. 1. Surface Evolver output files are parsed and processed [10] to access the complete data generated by the simulation. Our application works with any SE simulation (2D or $3 \mathrm{D}$ ) and no changes to the simulation output are necessary to accommodate the application. This processing addresses the "data access" challenge.

We visualize important simulation attributes which include bubble scalar measures and bubble velocity, location of topological changes and forces acting on solid objects interacting with foam. Overall foam behavior is analyzed using the average feature, kernel density estimate for topological changes and bubble paths. These visualization methods address the need of foam scientists to improve their understanding of the general foam behavior.

Domain experts wish to understand what triggers certain behavior in foam simulations by examining several simulation attributes. They also want to compare and contrast simulations with different parameters or different time steps of the same simulation. These requirements are addressed using multiple linkedviews. We can examine, in different views, different visualization attributes, time steps, visualization methods or simulations either two or three dimensional.

\subsection{Time-dependent visualizations}

Time-dependent visualizations are used for understanding general foam behavior. Visualization of bubble paths (Fig 3) provides information about the trajectory of individual bubbles in the simulation. The paths are a useful way to compare simulations with experiments. A bubble path is determined by connecting the position of a bubble's center over consecutive time steps.

A good way to smooth out variations caused by topological changes and to reveal general trends in data is to calculate the average (Fig. 4) of the simulation attributes over all time steps, or over a time window before the current time step. This visualization reveals global trends in the data because large fluctuations caused by topological changes are removed. This results in only small variations between averaged successive time steps. For foam simulations that include dynamic objects interacting with foam, we are interested in triggers to objects' behavior which are determined by foam properties around the objects. However, examining bubble attributes around objects for every time step is not always the best option. There is too much detail and bubble attribute values have large fluctuations caused by topological changes. To address this issue, we compute an average of attribute values around the dynamic objects using the approach of Lipşa et al. [10]. To compute the average of simulation attributes we run a one time preprocessing step that converts the unstructured grid simulation data into a regular grid and save the regular grid data on the hard-drive. This data is read each time we compute an average for a simulation attribute.

Foam topological changes are a manifestation of plasticity in foam. Domain experts expect that the $\mathrm{T} 1$ distribution will be an important tool for validating simulations against experiments and continuum models. Simply rendering the 
position of each topological change suffers from over-plotting, so it may paint a misleading picture of the real distribution. We compute a kernel density estimate $(K D E)$ [14] for topological changes. In foam simulation data, each topological change has two properties specifying when and where the topological change occurred. We place a Gaussian at each topological change position and we average these together. The standard deviation for the Gaussian is a user defined parameter which determines the amount of detail that is visible in the final visualization. Its initial value is one bubble diameter.

\subsection{Interaction}

Interaction with the data is an essential feature of our application. We provide the common navigation operations such as rotation, translation and scaling. We can select and/or filter bubbles and center paths based on three distinct criteria: based on bubble IDs, to enable relating to the simulation files and for debugging purposes; based on location of bubbles, to analyze interesting features at certain locations in the data; and based on an interval of attribute values specified using a histogram tool. A composite selection can be specified using both location and attribute values. To reveals features of interest in data we can change the color map used for displaying scalars or vectors or specify the range of values used in the color map through clamping.

\section{Case Studies}

We describe several examples in which our software is used to visualize foam simulation data. Our tool has been developed in close collaboration with domain experts who analyze the visualizations presented in these examples. For these case studies, we use two simulations: the falling disc (2D) and the falling sphere (3D) simulations. Our application, however, can process any Surface Evolver simulation. Both cases simulate a disc/sphere falling through a monodisperse (bubbles having equal volume) foam under gravity. In 2D we have 254 time steps and 1500 bubbles. In 3D we have 208 time steps and 144 bubbles. Note that the number of bubbles that we are able to simulate in 3D is severely restricted by the duration of the simulation. These simulations are relevant to industrial processes in mineral separation.

\subsection{Topological change trails for the falling disc (2D) and falling sphere (3D) simulations}

In a two-dimensional foam, a T1 occurs when two bubbles approach one another and two move apart. A bubble edge shrinks to zero length, forming an unstable vertex at which four edges meet. This is energetically unstable (Plateau's laws [3]), and immediately dissociates into two vertices separated by a new edge. The two bubbles that were initially neighbors move apart, and the two approaching bubbles become neighbors. We represent each of these events as a point on Fig. 2 left.

In a three-dimensional foam, the situation is more complicated. Bubbles have more degrees of freedom when they move, and there are different cases that we must consider. Firstly, there are two "standard" T1s: 


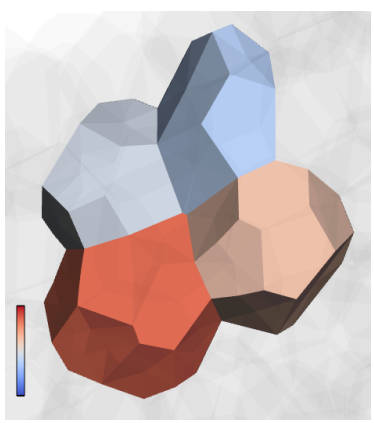

(a) $t=36$

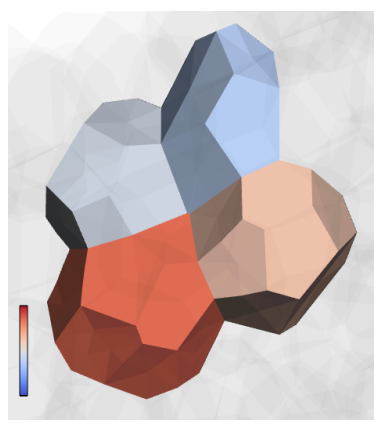

(b) $t=37$

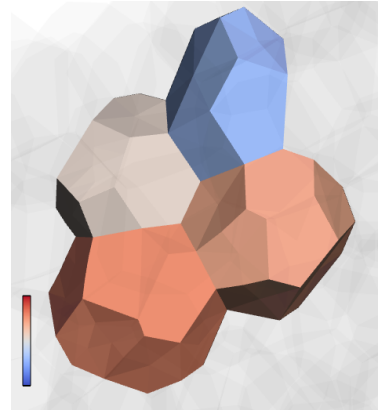

(c) $t=38$

Fig. 1: 3D topological change of type tri_to_edge. Bubbles are colored by number of faces per bubble: $(18,15,13,12)$. The first two images show bubbles just before the topological change and the third image shows bubbles after the topological change. After the topology change the number of faces in each bubble changes to $(17,16,14,11)$.

1. if a bubble edge shrinks to zero length in $3 \mathrm{D}$, then the resulting unstable vertex is replaced by a small triangular face (soap film); following Brakke [16], we refer to this as an edge_to_tri transition;

2. alternatively, if a small triangular face shrinks to zero area, then the resulting unstable vertex is replaced by a short edge; we refer to this as an tri_to_edge transition;

3. a further T1, in which a rectangular face shrinks to zero area and is replaced by another rectangular face, perpendicular to the first one, can be viewed as a composition of the above two topological changes; we refer to it as a quad_to_quad transition;

4. there are also two topological changes that we use to ensure that the topology of the tessellation remains an accurate representation of foam structure, for example if the structure is such that none of the above changes complete correctly: firstly, an edge may acquire more than three faces attached to it (violating another of Plateau's laws), in which case we perform a pop_edge transition to introduce a rectangular face;

5. secondly, a vertex may become attached to more than four edges (violating the 3D version of Plateau's first law), in which case we perform a pop_vertex transition to introduce a new edge joining two vertices.

We represent each of these T1s with a different color sphere, see e.g. Fig. 2 right. Fig 2 shows good agreement between the 2D and 3D datasets. Both simulations display a trail of T1s within close proximity of the path of the falling object. This demonstrates where the foam has been deformed the most, or "fluidized", by the influence of the solid object.

The disc in $2 \mathrm{D}$ seems to have a more wide ranging effect on the foam than in $3 \mathrm{D}$. This may be the result of the $3 \mathrm{D}$ foam being too small for a more fair comparison here. The 3D small sample means that the foam might be over constrained. The bubbles have nowhere to go out of the way of the sphere and will therefore just stay in front of the sphere and move with it. A surprising feature of the simulation discovered using our software is that there are no tri_to_edge topo- 

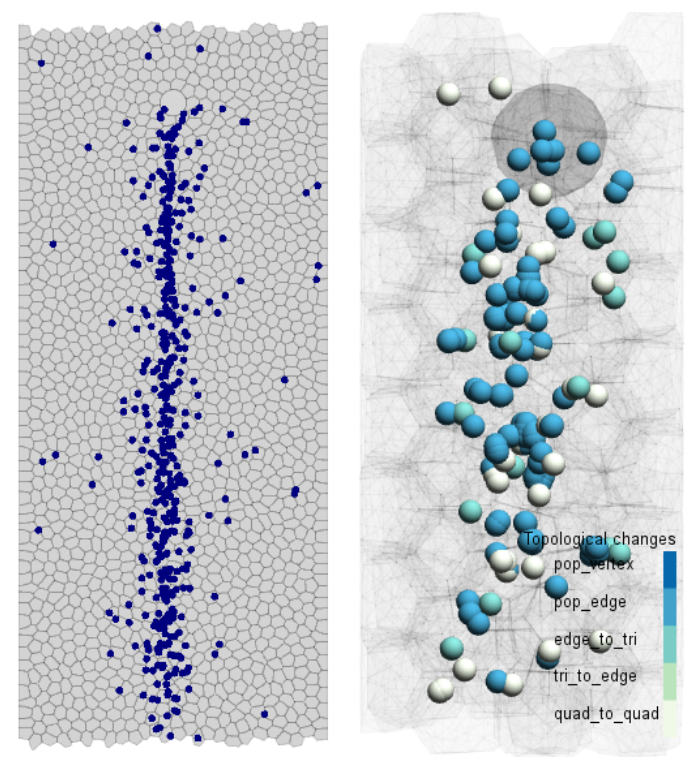

Fig. 2: Topological change trails for the falling disc (2D) and falling sphere (3D) simulations. In $3 \mathrm{D}$, topological changes are represented as spheres colored by the their type.

logical changes. Through investigation, domain experts realized that the order in which tests for deciding which different types of topological changes are applied matter. In particular, tri_to_edge and quad_to_quad types of topological changes are exclusive, you get one or the other depending on which you test first. Note this is a feature of the simulation, it is not known which types or what is the distribution of different types of topological changes that happen in real foam. These are interesting questions for future foam research.

\subsection{Bubble loops in 3D}

This visualization confirms for domain scientists that, as in $2 \mathrm{D}$, bubbles traverse loops in 3D in an axisymmetric way to provide space for the descending sphere. Fig. 3-right shows a bubble and the sphere paths color-mapped to velocity along the $Y$ axis, with blue showing downward velocity and red showing upward velocity. A loop consists of a downward segment (colored blue) and an upward loop (colored red). A bubble traverses the downward segment as the descending sphere approaches it. Then it traverses the upward loop as the sphere passes by it. The bubble avoids the falling sphere and then fills the space that it leaves. The loops get smaller as the distance of the bubbles to the sphere gets larger. A future direction of investigation for domain experts, triggered by our visualization, is to use the loop size to determine the distances to which the sphere influences the foam.

In Fig. 4 we see that essentially the same thing is happening both in $2 \mathrm{D}$ and 3D. For the 3D case, it is not quite as smooth due to the small size of the simulation. We see a circulation flow either side of the disc in 2D and all around the sphere in $3 \mathrm{D}$. This is the result of the volume constraint for both simulations. 


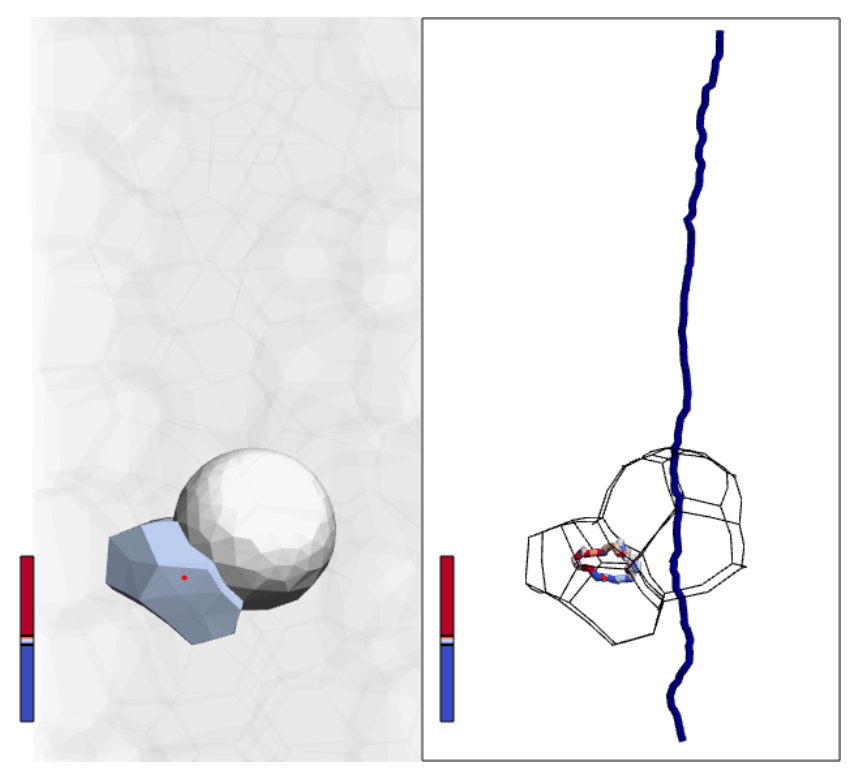

Fig. 3: A bubble path that forms a loop. This behavior was not previously observed in $3 \mathrm{D}$ by domain experts. The two views show the falling sphere and the bubble that traverses a loop. Bubble center is marked with a red dot. The right view shows only edges for the sphere and bubble and the paths traversed during the simulation. Bubble paths are colored by velocity along the $Y$ axis, with blue showing downward and red showing upward velocity.

\subsection{KDE for topological changes around the falling disc (2D) and falling sphere $(3 \mathrm{D})$ simulations}

Applying a KDE visualization for topological changes around the falling sphere yielded a surprising result: a density sphere centered just above the falling object, instead of the pear shape that we got for a 2D simulation of a falling disc (Fig 5b). We investigate possible causes and we discover that certain time steps have a large number of topological changes occurring approximately at the same position - on top of the falling sphere. Note that the maximum value in the color bar for 3D is 36 which denotes the maximum number of topological changes that occur in one time step. Repeated topological changes occurring on top of the falling sphere dominate the final result. These topological changes are an artefact of the quasistatic approximation, which allows faces or edges to repeatedly undergo a T1 and then a "reverse" T1 during convergence. Our collaborators investigate ways in which to eliminate this artefact, for example by introducing dissipation.

\subsection{Topological changes cause high velocity bubbles}

Previously, foam scientists hypothesized that high velocities are caused by topological changes (T1s) and we were able to verify that this is the case in 2D. We can now verify this hypothesis in 3D by matching T1 positions with positions of high velocity bubbles. The disordered directions of the arrows in Fig. 6 right is 


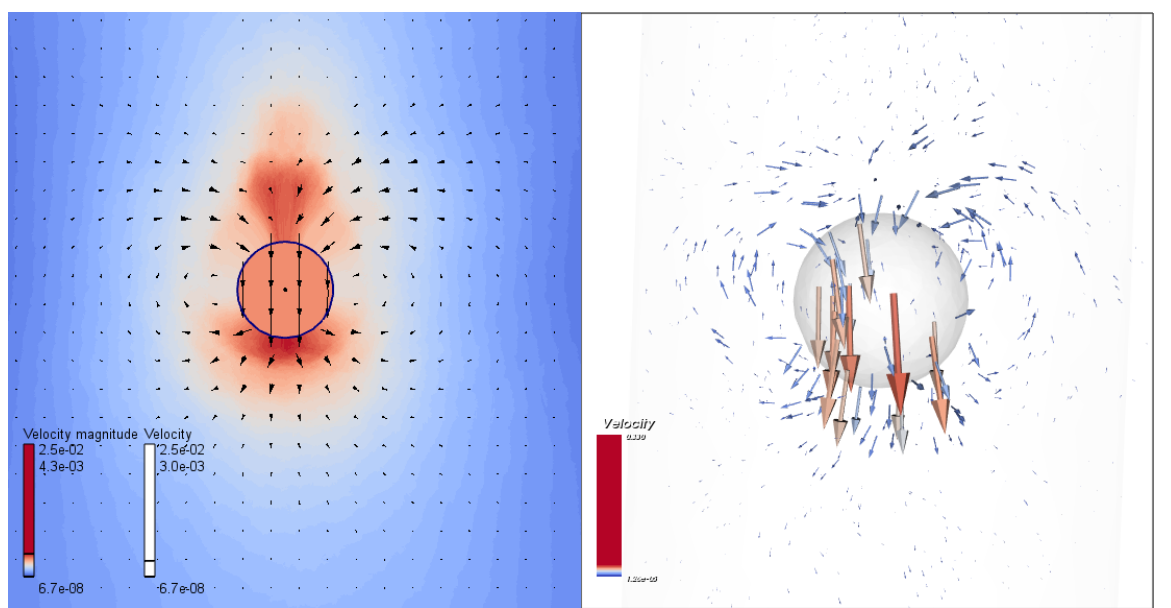

Fig. 4: Velocity average around the falling disc (2D) versus the falling sphere (3D) simulations. A similar pattern can be observed in 2D (left view) and 3D (right view). Bubbles are pushed down by the falling object, they move to the side to make space for it, and then they fill its space as the object passes them. In the left view we show velocity magnitude scalar and the velocity vector. In the right view we show the velocity vector colored by velocity magnitude. Both the scalar and vectors sizes are clamped using the color bars shown in the lower left corners.

a result of the topological change. Space left by bubbles moving away from each other close to the topological change (red arrows moving in opposite directions) is filled by bubbles in close proximity (smaller blue arrows pointing upwards).

\section{Conclusions and Future Work}

We describe foam research challenges and visualization solutions to address them. We present the first tool that enables interaction, visualization and analysis of 3D, time-dependent foam simulation data. We visualize scalar and vector simulation attributes as well as forces acting on objects in foam and position and type of topological changes. Time-dependent visualization include average of simulation attributes, KDE and bubble paths. Our tool validates previous hypothesis, offers means to debug simulations and helps finding new directions of research.

For future work, we would like to expand our tool to offer tensor and volume visualization and to support comparisons between simulations and experiments.

\section{Acknowledgments}

This research was supported in part by the Research Institute of Visual Computing (rivic.org) Wales. We thank Ken Brakke for answering our many questions about the Surface Evolver. ITD thanks Coleg Cymraeg Cenedlaethol for support. SC acknowledges financial support from the FP7 Marie Curie IAPP Project PIAP-GA-2009-251475-HYDROFRAC 

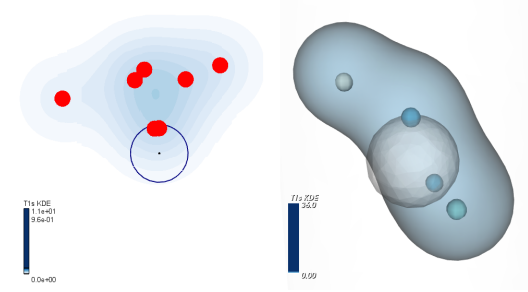

(a) KDE for one time step: $t=18$ left view and $t=21$ right view. Isosurface density is 0.5 for the right view.

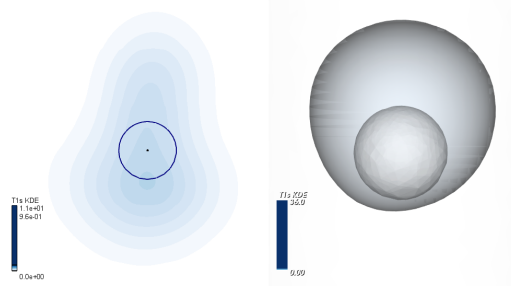

(b) KDE for all time steps. Isosurface density is 0.12 for the right view.

Fig. 5: KDE around the falling disc versus falling sphere simulations. The maximum values in the color bar represent the maximum number of topological changes in a time step. KDE for all time steps (b) shows that, for 3D, topological changes on top of the sphere dominate the final result. This is caused by topological changes in the same area being triggered repeatedly in the simulation code, feature discovered using our visualization.

\section{References}

1. J. Bigler, J. Guilkey, C. Gribble, C. Hansen, and S. Parker. A Case Study: Visualizing Material Point Method Data. EG Computer Graphics Forum, pages 299-306, 2006.

2. K. Brakke. The Surface Evolver. Experimental Mathematics, 1(2):141-165, 1992.

3. I. Cantat, S. Cohen-Addad, F. Elias, F. Graner, R. Höhler, O. Pitois, F. Rouyer, and A. Saint-Jalmes. Foams. Structure and Dynamics. Oxford University Press, 2013. Translated by Ruth Flatman, Edited by Simon Cox.

4. Y. Chen, B. Schaeffer, M. Weislogel, and G. Zimmerli. Introducing SE-FIT: Surface Evolver-Fluid Interface Tool for Studying Capillary Surfaces. In Proc. 49th AIAA Aerospace Sciences Meeting, pages 1-11, 2011. http://se-fit.com/.

5. S. Cox, D. Lipşa, I. Davies, and R. Laramee. Visualizing the dynamics of twodimensional foams with FoamVis. Colloids and Surfaces A: Physicochemical and Engineering Aspects, 2013. In press.

6. A. Glassner. Soap Bubbles: Part 1. Computer Graphics and Applications, IEEE, 20(5):76-84, Sep./Oct. 2000.

7. A. Glassner. Soap bubbles: Part 2 [computer graphics]. Computer Graphics and Applications, IEEE, 20(6):99 -109, Nov./Dec. 2000.

8. M. Hadwiger, F. Laura, C. Rezk-Salama, T. Höllt, G. Geier, and T. Pabel. Interactive Volume Exploration for Feature Detection and Quantification in Industrial CT Data. Visualization and Computer Graphics, IEEE Transactions on, 14(6):15071514, 2008.

9. A. König, H. Doleisch, A. Kottar, B. Kriszt, and E. Gröller. AlVis-An AluminiumFoam Visualization and Investigation Tool. In Visualization (VisSym), EG/IEEE TCVG Symposium on. Amsterdam, The Netherlands, 2000.

10. D. R. Lipşa, R. S. Laramee, S. J. Cox, and I. T. Davies. FoamVis: Visualization of 2D Foam Simulation Data. Visualization and Computer Graphics, IEEE Transactions on, 17(12):2096-2105, Oct. 2011.

11. D. R. Lipşa, R. S. Laramee, S. J. Cox, J. C. Roberts, R. Walker, M. A. Borkin, and H. Pfister. Visualization for the Physical Sciences. EG Computer Graphics Forum, 31(8):2317-2347, Dec. 2012. 

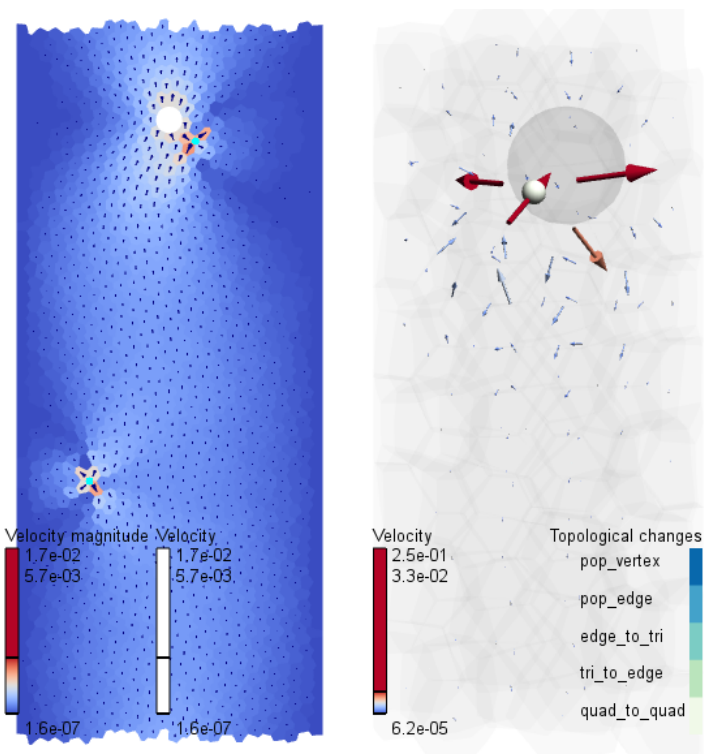

Fig. 6: Topological changes cause high velocity bubbles. In the left view we show bubble velocity and velocity magnitude scalar as well as the position of topological changes at $t=6$. In the right view we show bubble velocity colored by velocity magnitude and the position of topological changes at $t=12$. Note that in both views the velocity (and velocity magnitude) is clamped because velocities caused by topological changes are much larger than bubble velocities caused by the falling sphere.

12. F. Losasso, J. Talton, N. Kwatra, and R. Fedkiw. Two-Way Coupled SPH and Particle Level Set Fluid Simulation. Visualization and Computer Graphics, IEEE Transactions on, 14(4):797 -804, 2008.

13. R. Shimada, S. Rahman, and Y. Kawaguchi. Simulating the Coalescence and Separation of Bubble and Foam by Particle Level Set Method. In Computer Graphics, Imaging and Visualisation, 2008. CGIV'08. Fifth International Conference on, pages $18-22,2008$.

14. B. Silverman. Density Estimation for Statistics and Data Analysis, volume 26 of Monographs on Statistics and Applied Probability. Chapman \& Hall/CRC, 1986.

15. C. Smith. Grain shapes and other metallurgical applications of topology. In Metal Interfaces, pages 65-108. American Society for Metals, Cleveland, OH, 1952.

16. Surface Evolver Workshop, Apr. 2004. Online document, accessed 1 Dec. 2010, http://www.susqu.edu/brakke/evolver/workshop/workshop.htm.

17. R. Durikovič. Animation of Soap Bubble Dynamics, Cluster Formation and Collision. EG Computer Graphics Forum, 20(3):67-75, 2001.

18. D. Weaire and S. Hutzler. The Physics of Foams. Oxford University Press, Oxford, 1999. 